Psychology of Language and Communication 2013, Vol. 17, No. 3

VERSITAOPEN

DOI: $10.2478 /$ plc-2013-0019

\author{
TAKESHI KISHIMOTO \\ University of the Sacred Heart, Tokyo
}

\title{
DO REFERENTIAL PROBLEM SPACES AFFECT THE FREQUENCY OF IMPERATIVE POINTING BY INFANTS?
}

\begin{abstract}
Before speaking language, infants produce pointing gestures. To elucidate the mechanism promoting imperative pointing by infants, we investigated whether referential problem spaces, which are eco-cultural contexts in which subjects are reliant on others to obtain desirable but unreachable objects, affect the parental awareness of the frequency of imperative pointing by infants. Through a survey questionnaire, we asked parents of infants aged 8-30 months about the kinds of objects they place away from their infants and the frequency of their infants' imperative pointing. The results show that parents who mentioned placing tiny objects or objects mainly used by adults or older children away from their infants reported higher frequencies of imperative pointing by their infants than those who did not. This suggests that the frequency of infants' imperative pointing is increased by referential problem spaces, which are constructed by placing tiny objects or objects mainly used by adults or older children away from the infants.

Key words: imperative pointing, referential problem spaces, parents, infant, communication, parent-infant interaction
\end{abstract}

Although one-year-old infants cannot fully express what they want linguistically, they can do so through pointing gestures. Some previous studies have indicated that pointing gestures in infants start at an average age of 11 months (Butterworth, 2003), while others have suggested that infants begin using such gestures between the ages of 8-15 months (Carpenter, Nagell, \& Tomasello, 1998; Franco, 2005; Leung \& Rheingold, 1981; Liszkowski \& Tomasello, 2011). On the basis of previous studies, a distinction is often made between imperative and declarative pointing gestures. Infants use imperative pointing as a social tool to obtain objects (Bates, Camaioni, \& Volterra, 1975; Liszkowski, Carpenter, Striano, \& Tomasello, 2004). On the other hand, infants use declarative pointing

Address for correspondence: Takeshi Kishimoto, University of the Sacred Heart, 4-3-1 Hiroo, 11 Shibuyaku, Tokyo, 150-8938, Japan. E-mail: tkishimoto@u-sacred-heart.ac.jp 
to share their attention and interest in an object or event with adults (Liszkowski et al., 2004). Although it was demonstrated that infants come to be able to use imperative pointing earlier than declarative pointing (Camaioni, Perucchini, Bellagamba, \& Colonnesi, 2004), until today, little is known on how infants develop the imperative pointing.

Recently, Liszkowski, Brown, Callaghan, Takada, and De Vos (2012) demonstrated that infants around 1 year of age from various cultures produce pointing gestures at similar frequencies when the context was controlled. They used a standardized, semi-natural elicitation procedure in seven very different cultural environments around the world, which included Papua New Guinea, Indonesia, Japan, Peru, two regions in Mexico, and Canada. In their study, infants and their caregivers were observed for about 5 minutes in a room. Although there were many stimuli in the room, such as balloons or posters, the infants and their caregivers were asked not to touch them. Their results showed that by 10-14 months of age, the infants and their caregivers from various cultures pointed with similar frequencies and the same prototypical extension of the index finger in the same basic situation. This suggests that pointing gestures are acquired developmentally by infants via universal mechanisms inherent in daily caregiver-infant interactions. A candidate is the inhibition of infants' touching things by the caregivers, which was seen in the experiment by Liszkowski et al. (2012). Such inhibition by caregivers would promote their infants' imperative pointing gestures.

Research on the pointing gestures of chimpanzees (Pan troglodytes) has significant potential to support this idea. To date, only a few studies have observed the pointing gestures of chimpanzees in the wild (Tomasello, 2006). In contrast, some studies have suggested that captive chimpanzees frequently produce pointing gestures when they interact with human experimenters, even if the chimpanzees are not taught by humans how to point (e.g., Leavens, Hopkins, \& Bard, 1996). Leavens, Hopkins, and Bard (2005) claim that captive chimpanzees, unlike their counterparts in the wild, produce pointing gestures because of differences in their living environments. Chimpanzees in captivity often experience physical barriers (such as cages) to directly obtaining desirable but unreachable food and are provided with food by humans on a daily basis. This means that captive chimpanzees face a referential problem space, which is an eco-cultural context in which they are reliant on others to obtain food. Thus, pointing gestures by captive chimpanzees emerge as a problem-solving tactic to obtain desirable objects (in this case, food) by prompting humans to bring the objects to them. In contrast, chimpanzees in the wild do not produce pointing gestures, because they do not face a referential problem space that requires them to manipulate others in order to obtain something they want (Leavens \& Bard, 2011; Leavens et al., 2005). Similar to captive chimpanzees, human infants also often encounter a referential problem space owing to their 
immature locomotor capacities. Desirable objects for infants, such as candies, are often placed in unreachable locations and are delivered to them by their caretakers on a limited basis. To overcome the referential problem space and obtain these unreachable but desirable objects, infants resort to imperative pointing to direct their caretakers' attention to the objects and encourage their caretakers to deliver those objects to them. Thus, it is possible that such referential problem spaces promote infants' production of imperative pointing (Leavens \& Bard, 2011; Leavens et al., 2005).

The study by Cochet, Jover, and Vauclair (2011) supports this hypothesis. They successfully elicited pointing from 1-year-old infants by placing attractive toys beyond their reach. This study suggested that the production of imperative pointing by infants is promoted when the infants faced a referential problem space, i.e., that emerged between the infants and the unreachable toys. Although the study of Cochet et al. (2011) demonstrated that referential problem space affects the frequency of imperative pointing by infants, their study was conducted in a controlled experimental setting; it is therefore still unclear whether the referential problem spaces found in daily interactions between infants and caregivers are the same as those in Cochet et al's (2011) study. It is therefore possible that the referential problem spaces faced by infants in their daily lives do not affect the frequency of their imperative pointing.

Observing what kinds of objects are involved in the referential problem spaces faced by infants in their own homes is somewhat difficult, because each home differs in terms of the objects therein and their placement. There is substantial variation in such objects, and it would be complicated to investigate all possible objects in the limited timeframe of observation. Thus, in this study, we asked the parents of infants aged 8-30 months what kinds of objects they place in locations that are unreachable by their infants through administration of a questionnaire. Many previous studies have shown that parents provide reliable information on the communicative-linguistic development of their own children (e.g., Camaioni, Castelli, Longobardi, \& Volterra, 1991). Furthermore, questionnaire administration enabled us to obtain a large sample, which would not have otherwise been possible, and which enabled us to assemble a wealth of information on the objects that construct the referential problem spaces faced by infants in their homes. Thus, by this procedure, we measured the parental awareness of the frequency of imperative pointing by their infants as the estimate of the actual frequency of imperative pointing by their infants, and the objects that construct the referential problem spaces faced by infants in their homes.

Although Leavens et al. (2005) noted that chimpanzees face referential problem spaces constructed by food in unreachable places, human infants may face referential problem spaces constructed by unreachable objects other than food. For example, parents often distance their infants from dangerous or undesirable objects, such as knives, scissors, and trash cans; tiny objects which infants may 
accidentally ingest, such as beads, drugs, and sewing needles; and objects often used by adults or older children, such as personal computers, important documents, and tissue boxes. Thus, we sorted the unreachable objects mentioned by parents into four categories: food, dangerous objects that may injure infants, tiny objects that may be accidentally ingested by infants, and objects mainly used by adults or older children. On the basis of these responses, we estimated which kinds of unreachable objects might construct the referential problem spaces for infants. In the same questionnaire, we also asked the parents about the frequency of imperative pointing by their infants and analyzed whether referential problem spaces constructed by the unreachable objects mentioned above affect the frequency of the infants' imperative pointing.

In this study, we investigated whether the production of imperative pointing gestures by infants was affected by the existence of referential problem spaces in the infants' home. If the production of imperative pointing by infants were affected by the existence of referential problem spaces, the parents who place objects away from their infants would report the imperative pointing by their infants more frequently than those who do not, even after controlling the age of the infants. The lower age limit of the infants was set as 8 months on the basis of evidence indicating that pointing gestures emerge beginning at this age (Liszkowski \& Tomasello, 2011). Furthermore, the upper age limit of the questionees' infants was set as 30 months because the children's communication is still reliant to a large degree on nonverbal action (Grosse, Behne, \& Tomasello, 2010).

\section{Method}

\section{Participants}

The participants in this study were parents and infants living around Tokyo, Japan. Questionnaires were distributed to at least 254 parents from two kindergartens and seven nursery schools. Of these, 158 were returned (collection rate: $62.2 \%) .{ }^{1}$ The research was conducted with fully informed permission from the Research Ethics Committee of the Department of Psychology, University of the Sacred Heart.

Among the potential respondents, 45 were eliminated: 16 were eliminated because of incomplete forms, 10 because the infants were below the lower age limit of the study (i.e., under 8 months old), and 19 because the infants were above the upper age limit of the study (i.e., over 30 months old). Thus, the final sample included 113 respondents. Among the 113 respondents, 110 (97.3\%) were mothers, and only $3(2.7 \%)$ were fathers. The average age of the respondents was 34.4 (range: 23-44; standard deviation: 4.34). Twenty-two (19.9\%) respondents were

\footnotetext{
${ }^{1}$ Since additional questionnaires were also distributed by the staff members of the nursery schools, it is possible that the collection rate was slightly lower than this.
} 
full-time homemakers, 15 (13.4\%) were part-timers, 70 (61.9\%) was full-timers, and for $6(5.3 \%)$ we did not have data. ${ }^{2}$

In this study, we asked parents to answer the questions about their youngest or only child. Hereafter, we refer to the infants being studied as the "target children." The target children were comprised by 70 boys and 43 girls. The mean age of the target children was 20.5 months (range: 8-30 months; standard deviation: 6.49). There were no significant differences in mean age between the boys and the girls (21.1 and 19.5 months; $t(111)=1.32$, 4n.s.).

\section{Measurement}

We asked parents to answer the following questions ${ }^{3}$ about their target children:

(1) Dates when parents answered the questionnaire and birthdays of the target children. To determine the correct ages (in months) of the target children, we asked parents the dates on which they answered the questionnaires and on which their children were born.

(2) Objects the parents placed in locations that were unreachable by their infants. To determine the kinds of objects that construct the referential problem spaces for the infants, we asked the parents which objects they place away (i.e. out of the reach or sight) from their infants. This question was open-ended, enabling the parents to freely write as many objects as they could remember or to leave the question blank if they did not place any objects in unreachable locations.

(3) Frequency of imperative pointing by the target children.

To examine the frequency of the target children's imperative pointing gestures, the parents were asked how often their children had made pointing gestures to request that the parents retrieve what they wanted within the past week. The frequency was evaluated using a 4-point scale: 1 (never), 2 (1-2 times per day), 3 (3-4 times per day), and 4 ( $\geq 5$ times per day). ${ }^{4}$

\footnotetext{
${ }^{2}$ We could not know the precise time the participants spent with their infants. However, the data about the average time the mothers living in Tokyo spend in caring (e.g. playing with or monitoring) their child (under 6 years old) exists (Ministry of Internal Affairs and Communications, 2012). From the data, we can find that the mothers who had jobs spent 2 hours and 33 minutes per day in caring their child, and who did not have jobs spent 3 hours and 26 minutes per day. Although it is suggested that the time spent with infants differ among the parents' employment formats, there were no significant differences in the parental reports of the frequency of the imperative pointing by their infants among four employment formats in this study (e.g. full-time homemakers, part-timers, full-timers, and unknown) (Kluskal-Wallis test, $\chi^{2}=7.11, d f=3$, n.s.).

${ }^{3}$ We asked several other questions, including some regarding the locomotive patterns of the target children. Findings regarding the relationship between the locomotive patterns of target children and the frequency of their pointing gestures have been reported in a study by Kishimoto (2011). The participants of this study were from the same participants pool of Kishimoto (2011). However, the analyses conducted in this manuscript are entirely original.

${ }^{4}$ We were afraid that it would be difficult for parents to accurately render the precise frequency of imperative pointing by their target children. To ascertain the frequency of infants' pointing gestures
} 
There was a possibility that parents could speculate regarding the researcher's expectations while answering the questionnaire. To prevent this, the parents were not told the goal of the study (i.e., to investigate the relationship between the frequency of imperative pointing by infants and the existence of referential problem spaces) but were told that the questionnaire was conducted in order to reveal how caregivers living with infants think and behave in their daily lives.

\section{Coding and Analysis}

A total of 309 objects were identified by the parents as having been placed in locations that were unreachable by their infants. We coded these objects into the following four categories:

(1) food, including items eaten by infants daily at appropriate times (e.g., bread, fruit, or candy);

(2) dangerous objects that can injure or contaminate infants, including those that can hurt infants if they fall to the floor while holding the objects in their hands (e.g., knife, trash can, or ball-point pen);

(3) tiny objects that the infants can accidentally ingest (e.g., batteries, beads, or sewing needles); and

(4) objects mainly used by adults or older children (e.g., personal computer, TV remote, or tissue box).

Twenty percent (62) of the objects were randomly selected and coded by a graduate student who was not informed of the aim of this study. The Cohen's kappa value for this coding procedure was 0.78 , which means that the inter-coder reliability was substantial (Fleiss, Levin, \& Paik, 2003).

We used ordinal logistic regression to assess the influence of the presence of the objects categorized above on the frequency of imperative pointing by the infants. Logistic regression fits a linear model to the logit (log odds) of a discrete dependent variable. The coefficient $\beta$ is given in terms of the log odds; therefore, $\exp (\beta)$ gives the change in odds per unit change in the associated predictor (DeScioli \& Kurzban, 2009). When the dependent variable has more than two categories and the categories contain ordinal information, as is the case in our study, ordinal logistic regression is appropriate. In this case, $\exp (\beta)$ describes the change in the generalized odds of being in a higher category on the ordinal scale (DeScioli \& Kurzban, 2009).

The dependent variable used in the analysis was the frequency of imperative pointing of the target children, as reported by their parents. The independent

on the basis of parent questionnaires, Camaioni et al. (1991) used a 3-point scale with different frequencies ("never," "sometimes," and "often") as the answer choices. Although it would be easy for parents to estimate the frequency of infants' pointing gestures on this 3-point scale, it was possible that interpretation of the meanings of "sometimes" and/or "often" would differ among parents. Inspired by the study of Camaioni et al. (2004), who had parents give the frequency of their children's pointing gestures as "1-2 times" or "several times," we used a 4-point scale with different frequencies ("never," "1-2 times per day," "3-4 times per day," and "5 or more times per day") as answer choices. 
variables entered into the ordinal logistic regression were age (in months) of the target children, gender (dummy variable; 0: boy; 1: girl) of the target children, and the objects the parents placed in unreachable locations from their children. We found that some objects mentioned by the parents were not countable. For example, some parents answered "tiny things that can be swallowed by children" instead of the names of concrete objects. Other parents mentioned objects that consist of multiple parts (e.g., audio equipment, including DVD players). We could not count the precise number of objects from these responses, but we did determine which types of objects were placed out of reach of children. Thus, we did not determine the specific numbers of objects belonging to each of the four categories; rather, we coded responses as " 1 " if the response mentioned objects in a certain category and "0" otherwise. For example, if a parent wrote "knife," "trash can," and "sewing needle," we coded the response as follows: "food: 0 , dangerous objects: 1 , tiny objects: 1 , and objects mainly used by adults or older children: 0 ." These object variables were treated as dummy variables in the ordinal logistic regressions.

The ordinal logistic regressions were conducted using the PASW statistics version 18 software package. ${ }^{5}$ The statistical significance of the resulting test statistics was examined via two-tailed tests in which the level of significance was set at $p<0.05$.

\section{Results}

\section{Kinds of Objects Moved Away from the Target Children}

First, we determined the number of parents who identified objects belonging to each category. Forty-seven out of 113 parents (41.6\%) mentioned food, 70 $(61.9 \%)$ mentioned dangerous objects, 61 (54.0\%) mentioned tiny objects, and $68(60.2 \%)$ mentioned objects mainly used by adults or older children. Eight of 113 parents $(7.1 \%)$ mentioned none of the four categories, $25(22.1 \%)$ mentioned one category, $31(27.4 \%)$ mentioned two categories, $37(32.7 \%)$ mentioned three categories, and $12(10.6 \%)$ mentioned all four categories.

Logistic regression results revealed the effect for the age of the target children on whether or not parents mentioned food (model $\left.\chi^{2}=4.10, p<0.05\right)$. The higher the age of the target children was, the higher the percentage of parents mentioned food ( $\beta=0.061$, Wald's $\left.\chi^{2}=3.954, d f=1, p<0.05\right)$. On the other hand, there were no effects of the age of the target children on whether or not parents mentioned dangerous objects (model $\chi^{2}=0.29$, n.s.), tiny objects (model $\chi^{2}=3.12$, n.s.), and objects mainly used by adults or older siblings (model $\chi^{2}=0.15$, n.s.).

\footnotetext{
${ }^{5}$ The ordinal logistic regression was conducted using the PASW software package using a generalized linear model (GLM) with a multinomial error structure and a cumulative logit link function.
} 
Table 1. Factors affecting the frequency of pointing in the target children, as reported by their parents

\begin{tabular}{|c|c|c|c|}
\hline Independent variable & $\beta$ (s.e.) & $d f$ & Wald $\chi^{2}$ \\
\hline Age (in month) of the target children & $0.111(0.033)$ & 1 & $11.02^{* *}$ \\
\hline Gender of the target children ${ }^{\mathrm{a}}$ & $0.448(0.420)$ & & \\
\hline \multicolumn{4}{|c|}{ Objects placed away from the target children ${ }^{\mathrm{b}}$} \\
\hline Food & $0.564(0.442)$ & 1 & 1.629 \\
\hline Dangerous objects & $0.124(0.407)$ & 1 & 0.093 \\
\hline Tiny objects & $1.003(0.407)$ & 1 & $6.064^{*}$ \\
\hline Objects mainly used by adults & $0.998(0.410)$ & 1 & $5.923^{*}$ \\
\hline
\end{tabular}

${ }^{* *} p<0.01 ;{ }^{*} p<0.05$

\footnotetext{
a The gender of the target children was treated as dummy variables and equal "0" when the target child was boy, "1" when the target child was girl.

b The four categories of objects were treated as dummy variables and equal " 1 " when an object in a category was mentioned and "0" otherwise.
}

\section{Frequency of Imperative Pointing by the Target Children}

The frequency of imperative pointing by the target children was positively associated with the age of the target children (Table 1) and with the presence or absence of tiny objects and objects mainly used by adults or older children. Parents who mentioned placing tiny objects away from the target children reported a higher frequency of imperative pointing by their children than those who did not $\left(\beta=1.003\right.$, Wald's $\left.\chi^{2}=6.064, d f=1, p<0.05\right)$. Similarly, parents who mentioned placing objects mainly used by adults or older children away from the target children reported a higher frequency of imperative pointing than those who did not $\left(\beta=0.998\right.$, Wald's $\left.\chi^{2}=5.923, d f=1, p<0.05\right)$ (Figure 1$)$. On the other hand, the frequency of imperative pointing by the target children was not associated with the gender of the target children, and the presence vs. absence of food or dangerous objects (Table 1, Figure 1).

\section{Discussion}

In this study, we investigated whether the frequency of infants' imperative pointing was affected by the presence vs. absence of objects placed in unreachable locations by them. The results indicated that parents who mentioned placing tiny objects or objects mainly used by adults or older children away from their infants reported a higher frequency of imperative pointing than those who did 

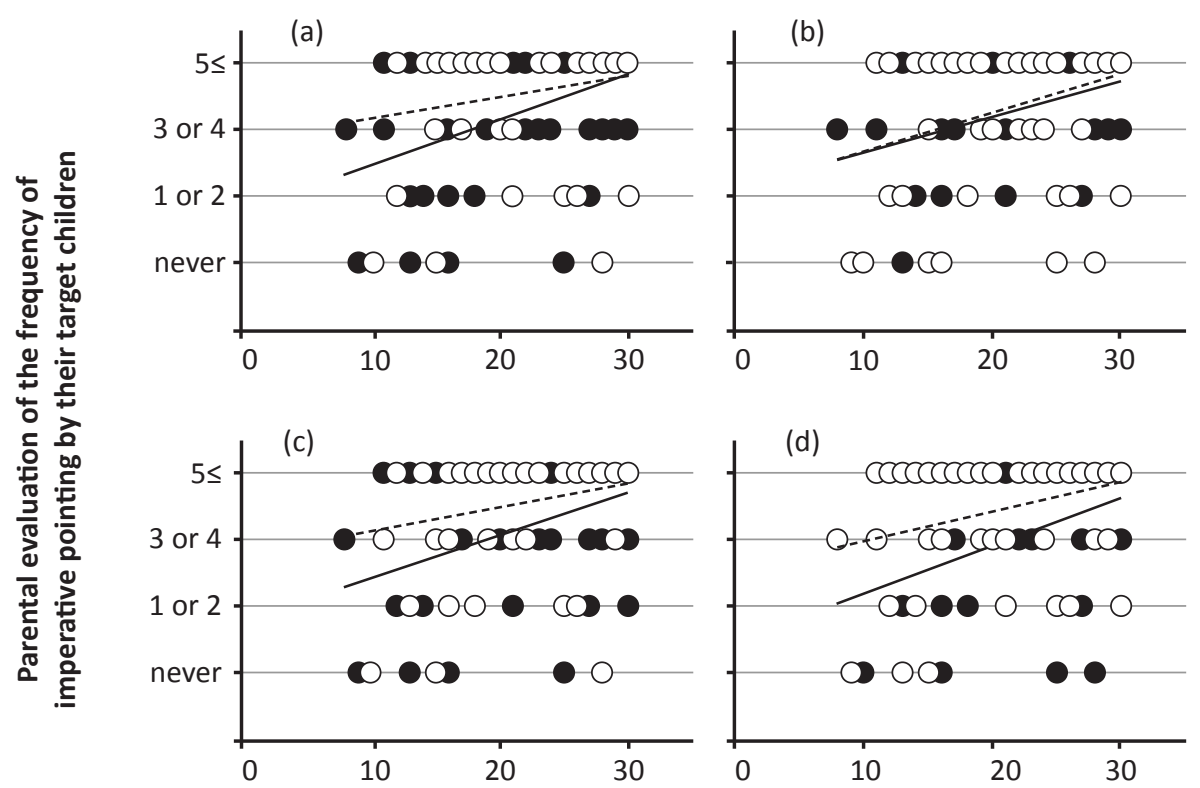

Age (in month) of the target children

Figure 1. The bivariate plot of the age (in months) of the target children (x-axis) against the frequency of their imperative pointing (y-axis), as reported by the parents who mentioned placing objects from the following categories away from their children: (a) food, (b) dangerous objects, (c) tiny objects, and (d) objects mainly used by adults or older children; the white dots represent the parents who mentioned placing objects from any of the above categories away from their children, while the black dots represent those who did not; the dashed line represents the best-fit line of the white dots, while the solid line represents the best-fit line of the black dots

not, as shown in Figure 1. This suggests that the frequency of imperative pointing by infants is promoted by referential problem spaces, which are constructed by placing tiny objects or objects mainly used by adults or older children away from infants. This is consistent with previous research on referential problem spaces and the imperative pointing gestures of chimpanzees and infants. Some studies have revealed that pointing gestures by chimpanzees are promoted by the presence of human experimenters and the existence of referential problem spaces such as that resulting from being in a mesh cage that prevents them from obtaining food (Leavens et al., 1996). Similarly, previous studies also revealed that human infants produce more imperative pointing in the presence of unreachable objects, suggesting that infants' imperative pointing is also promoted by 
referential problem spaces (Cochet et al., 2011). However, in contrast to these previous studies, which were mostly conducted in controlled environments, ours is the first to demonstrate that referential problem spaces encountered by infants in their daily lives affect imperative pointing. We suggested that the frequency of infants' imperative pointing was promoted by the everyday affairs which the caregivers place what they do not want their infants to touch away from their infants.

Although previous studies have suggested that referential problem spaces promote pointing by both chimpanzees and human infants, we demonstrated that the kinds of objects that promote imperative pointing by constructing referential problem spaces for chimpanzees and for humans vary. While pointing by chimpanzees was promoted when food was placed away from them (Leavens et al., 2005), our findings suggest that imperative pointing by infants was not promoted by food located in unreachable places but rather by tiny objects or objects mainly used by adults or older children. This result may have occurred because human infants are more attracted to tiny objects or objects mainly used by adults or older children than they are to food, in contrast to the chimpanzees, who may be more attracted to food.

Although parents often place tiny objects such as beads and objects mainly used by adults or older children such as personal computers away from infants, it is possible that they may sometimes allow infants to use or play with such objects under strict supervision. For example, parents may use personal computers with their infants. The infants' experience in using such attractive objects may prompt them to desire to repeat such use. However, because parents cannot always monitor what their infants do, they may move those objects away from their infants. Thus, infants may request those objects from their parents through imperative pointing. We speculate that in any given culture, infants are allowed to play with certain objects only under their caretakers' supervision; if their caregivers are busy or cannot monitor them, such objects are then moved away from the infants. Such circumstances thus construct the referential problem spaces that infants face in their daily lives, which in turn promote imperative pointing. On the other hand, perhaps the food or dangerous objects are under a more strict parental control thus these objects would not affect the frequency of imperative pointing by infants even if they were placed away from infants. Although we did not determine the precise processes by which infants acquire imperative pointing, our results suggested the common existence of the environmental conditions necessary for imperative pointing to emerge.

Because this study was conducted by administering a questionnaire and not by observing the interaction between infants and parents directly, there may be major problems to overcome in order to validate the interpretation of this study's results. One problem is that we did not validate the questionnaire used in this study, especially the question about the frequency of the children's imperative 
pointing. Figure 1 indicates that there might be a ceiling effect, as many responses clustered on " $\geq 5$ instances of pointing per day," suggesting that the question about the frequency of children's imperative pointing might be inappropriate. To solve this problem, it might be useful to create additional categories, such as " $5-10$ times per day" and " $\geq 10$ times per day," in future research. Additionally, we did not collect some important indexes which could have affected the production of pointing gestures by infants, such as the index of the infants' language development and SES. In the previous studies (Cochet, et al., 2011; Rowe \& Goldin-Meadow, 2009), both indexes are suggested to have effects on the infants' production of the pointing gestures. Future studies should ask these indexes and analyze the interactions of these variables and those in our study to understand the developmental mechanism of imperative pointing more precisely. Furthermore, the results indicating that referential problem spaces increase the frequency of infants' imperative pointing gestures could be obtained for different reasons than those mentioned above. One possibility is that the observations of the parents who answered this questionnaire mediated the relationship between the frequency of imperative pointing gestures by infants and the objects placed away from them. It is possible that parents who are careful enough to place tiny objects or objects mainly used by adults or older children away from their infants might also be more careful to monitor the imperative pointing gestures produced by their infants. Thus, the frequencies of infants' imperative pointing in this study may not necessarily reflect the actual frequencies, but rather the general higher awareness of the parents about their children's behavior. Because the design of this study was correlational, the results of this study should be interpreted cautiously. Our findings may therefore be confirmed in future studies by directly observing infant-caregiver interactions in their daily lives.

In conclusion, we found that parents who placed tiny objects or objects mainly used by adults or older children in unreachable places for their infants reported a higher frequency of imperative pointing among their target infants. This is the first study to suggest that imperative pointing by infants is promoted by the referential problem spaces that are constructed by unreachable objects in their daily lives. The pointing gestures are one of the commonly-used gestures, and the gestures are likely to be acquired spontaneously. However, we have suggested that the development of the pointing would be related to the environment which the desired objects were placed where the infants could not reach without the help of the parents.

\section{Acknowledgments}

I would like to thank Kiyobumi Kawakami, Keisuke Yazawa, Keiko Sakota, Keiko Teramura, Kimiko Yomogi, Keiko Watanabe, Tomoko Maruyama, Rika Kono, and all the staff members and kindergarten teachers of the participating 
nursery schools for permitting me to conduct this study and for their immense cooperation. I would also like to thank all the parents and their infants who participated in the study. This study has been supported by a MEXT KAKENHI (Grant-in-Aid no. 21830108) from the Ministry of Education, Culture, Sports, Science and Technology of the Japanese government.

\section{References}

Bates, E., Camaioni, L., \& Volterra, V. (1975). The acquisition of performatives prior to speech. Merrill-Palmer Quarterly, 21(3), 205-226.

Butterworth, G. (2003). Pointing is the royal road to language for babies. In S. Kita (Ed.), Pointing: Where Language, Culture, and Cognition Meet (pp. 9-33). Mahwah: Erlbaum.

Camaioni, L., Castelli, M.C., Longobardi, E., \& Volterra, V. (1991). A parent report instrument for early language assessment. First Language, 11(33), 345-359.

Camaioni, L., Perucchini, P., Bellagamba, F., \& Colonnesi, C. (2004). The role of declarative pointing in developing a theory of mind. Infancy, 5(3), 299-308.

Carpenter, M., Nagell, K., \& Tomasello, M. (1998). Social cognition, joint attention, and communicative competence from 9 to 15 months of age. Monographs of the Society for Research in Child Development, 63(4), 1-174.

Cochet, H., Jover, M., \& Vauclair, J. (2011). Hand preference for pointing gestures and bimanual manipulation around the vocabulary spurt period. fournal of Experimental Child Psychology, 110(3), 393-407.

DeScioli, P. \& Kurzban, R. (2009). The alliance hypothesis for human friendship. PLoS ONE, 4(6), e5802.

Fleiss J., Levin, B., \& Paik, M. (2003). Statistical Methods for Rates and Proportions (3rd ed.). Hoboken: Wiley \& Sons.

Franco, F. (2005). Infant pointing: Harlequin, servant of two masters. In N. Eilan, C. Hoerl, T. McCormack, \& J. Roessler (Eds.), Foint Attention: Communication and Other Minds. Problems in Philosophy and Psychology (pp. 129-164). Oxford: Oxford University Press.

Grosse, G., Behne, T., Carpenter, M., \& Tomasello, M. (2010). Infants communicate in order to be understood. Developmental Psychology, 46 (6), 1710-1722.

Kishimoto, T. (2011). Does infants' locomotive development affect parental awareness of pointing gestures by infants? Journal of Human Environmental Studies, 9(2), 77-82.

Leavens, D.A. \& Bard, K.A. (2011). Environmental influences on joint attention in great apes: Implications for human cognition. Fournal of Cognitive Education and Psychology, 10(1), 9-31.

Leavens, D.A., Hopkins, W.D., \& Bard, K.A. (1996). Indexical and referential pointing in chimpanzees (pan troglodytes). Journal of Comparative Psychology, 110(4), 346-353. 
Leavens, D.A., Hopkins, W.D., \& Bard, K.A. (2005). Understanding the point of chimpanzee pointing: Epigenesis and ecological validity. Current Directions in Psychological Science, 14(4), 185-189.

Leung, E.H.L., \& Rheingold, H.L. (1981). Development of pointing as a social gesture. Developmental Psychology, 17(2), 215-220.

Liszkowski, U., Brown, P., Callaghan, T., Takada, A., \& De Vos, C. (2012). A prelinguistic gestural universal of human communication. Cognitive Science, 36(4), 698-713.

Liszkowski, U., Carpenter, M., Henning, A., Striano, T., \& Tomasello, M. (2004). Twelve-month-olds point to share attention and interest. Developmental Science, 7(3), 9297-9307.

Liszkowski, U. \& Tomasello, M. (2011). Individual differences in social, cognitive, and morphological aspects of infant pointing. Cognitive Development, 26, 16-29.

Ministry of Internal Affairs and Communications (2012). 2011 Survey on Time Use and Leisure Activities > Questionaire A > Results on Time Use $>$ Time Use for Prefecture, Table No 2; Tokyo-to. Statistics Bureau, Ministry of Internal Affairs and Communications, September 26th, 2012. Retrieved 7.12.2013 from: http://www.e-stat.go.jp/SG1/estat/ListE.do?bid=000001040667\&cycode $=0$.

Tomasello, M. (2006). Why don't apes point? In N. Enfield \& S. Levinson (Eds.), Roots of Human Sociality: Culture, Cognition and Interaction (pp. 506-524). New York: Berg. 